\title{
Stable Growth of Large-area Single Crystalline Thin Films from an Organic Semiconductor/Polymer Blend Solution for High-mobility Organic Field-effect Transistors
}

Junshi Soeda $^{\mathrm{a}, \mathrm{b}}$, Toshihiro Okamoto ${ }^{\mathrm{a}, \mathrm{c}, *}$ Chikahiko Mitsui $^{\mathrm{a}}$, and Jun Takeya ${ }^{\mathrm{a}, \mathrm{b}, *}$

${ }^{a}$ Department of Advanced Materials Science, Graduate School of Frontier Sciences, The University of Tokyo, 5-1-5 Kashiwanoha, Kashiwa, Chiba 277-8561, Japan

${ }^{\mathrm{b}}$ Department of Applied Physics, Graduate School of Engineering, Osaka University

1-1 Yamadaoka, Suita, Osaka 567-0047, Japan

${ }^{\text {c }}$ PRESTO, JST, 4-1-8 Honcho, Kawaguchi, Saitama 332-0012, Japan.

* Corresponding authors: Toshihiro Okamoto and Jun Takeya

E-mail: tokamoto@k.u-tokyo.ac.jp; takeya@k.u-tokyo.ac.jp

Keywords: organic semiconductor/polymer blend; single crystalline thin film; organic field-effect transistor; large-area

\begin{abstract}
We developed an effective and steady solution-processing technique for a small moleculetype semiconductor, $\mathbf{C}_{\mathbf{1 0}}$-DNBDT-NW, by adding an amorphous PMMA polymer to produce stable growth of a two-dimensional large-area single-crystalline thin film by effective phase separation at a crucially faster processing speed compared to the case without the addition of a polymer. By using this solution-processing technique, it is noteworthy that the single-crystalline films of $\mathbf{C}_{\mathbf{1 0}}$-DNBDT-NW / PMMA exhibit the highest and average mobilities of 17 and $10.6 \mathrm{~cm}^{2} / \mathrm{Vs}$, respectively. Furthermore, we also show the limitations of two-dimensional continuous growth of a single-crystalline film in terms of the solution technique.
\end{abstract}




\section{Introduction}

A major challenge in printed electronics technology is reproducibly building homogeneous large-area organic semiconductor thin films with charge carrier mobilities. Such technology will have a dramatic effect; it should open novel electronics markets based on organic field-effect transistors (OFETs), which are anticipated to be next-generation low-cost, flexible, light-weight, and large-area switching electronic components.[1-5] Consequently, reliable solution-processing techniques are highly desired toward high-speed device applications such as radio-frequency identification (RF-ID) tags and gate-driver circuits of active matrices necessary for fully flexible displays.[6]

Among the various solution-processing techniques, one of the most common in the field of organic electronics is the spin-coating method, which can easily form thin films. However, spin-coating has some drawbacks; one is that a large amount of compounds is required because most of the semiconductor solution is spun-out and wasted. Another is that the polycrystalline films are randomly ordered in a radial fashion from the center area because organic semiconductors rapidly crystallize. These limitations result in a poor carrier mobility compared to the intrinsic mobility of organic semiconductors due to the inevitable crystal boundaries and interfacial traps, which are crucial for a high carrier mobility.[7, 8]

To reduce the amount of required materials and to grow crystalline thin films without grain boundaries, several recent approaches have focused on the development of promising solution processing techniques such as the double inkjet method[9] and the solution-shearing method[10] to grow single crystalline thin films with ordered and grain boundary-less natures.[11] These works have demonstrated the intrinsic high carrier mobility of organic semiconductors compared to less ordered cases. Our research group has developed a simple and easy solution-processing technique with minimal materials called the "edge-casting method".[12] In our method, material thin films are prepared from a solution, including a semiconductor in the controlled direction using the edge structure, resulting in a highly 
uniform and highly crystallized single crystalline thin film that exhibits an intrinsic carrier mobility.

Very recently, we developed a continuous growth method of single crystalline thin films by the edge-casting method, called "continuous edge-casting method", toward practical large-area device applications.[13] Employing this method, we obtained a highly ordered inch-sized single crystalline film using a high performance 3,11-didecyldinaphtho[2,3-d:2',3'-d']benzo[1,2-b:4,5-b']dithiophene $\left(\mathrm{C}_{10}-\mathrm{DNBDT}-\mathrm{NW}\right)$ organic semiconductor.[14] We also demonstrated transistor arrays with 25 transistors where the average carrier mobility reached $7.5 \mathrm{~cm}^{2} / \mathrm{Vs}$, indicating that the device performance is suitable for high-speed logic circuit applications.[13] However, this method has some drawbacks because single crystalline thin films are only obtained under optimal growth conditions in the representative parameters of the concentration of organic semiconductor solution, substrate moving speed, and substrate temperature, as illustrated in Figure 1a. The difficulty in controlling single-crystal growth of small molecule-type compounds from a solution due to natural phenomenon has motivated us not only to develop more reliable and reproducible solution-processing techniques, but also to clarify the size limitations for growing large-area and high-quality single-crystalline films suitable for high-speed organic semiconductors TFTs using low-cost and high-throughput large-area printing.

Figure 1a illustrates the concept of the continuous edge-casting method.[13] It has the following steps: 1) the semiconductor solution is supplied to the edge of the solution holding a blade. Simultaneously, the substrate on a hot plate at a certain temperature is moved toward the other side of the solution supply, which is depicted with an arrow. 2) The crystal nucleus emerges from the area of the contact line and then the crystalline film grows gradually to form an inch-sized single-crystalline film.

The most appropriate speed to form a smooth single-crystalline thin film of $\mathbf{C}_{10}-$ DNBDT-NW is reported to be $30 \mu \mathrm{m} / \mathrm{s}$. A faster processing speed results in poor ordering 
and discontinuous single-crystalline thin films as described in Table 1 (the pictures of the upper row). The result indicates that single-crystalline thin films spread two-dimensionally due to a very narrow processing window in the reported processing conditions. Because a subtle change in the growth conditions has a drastic impact on film morphology, it is essential to maintain the appropriate processing conditions.

The formation of the poor ordering and a discontinuous single-crystalline thin film at a faster speed prompted us to investigate the mechanism of crystal growth in the continuous edge-casting method from the viewpoints of "crystal growth" and "substrate moving" using three cases. In case (1), crystal growth and the substrate move at the same speeds to realize single-crystalline film grow continuously and two-dimensionally. In case (2), crystal growth has a faster speed and the solution sticks to the crystals grown on the substrate. In case (3), the substrate moves faster, preventing the semiconductor solution from sticking to the crystals, and the shape of the solution in the contact region starts deforming, as illustrated in Figure $1 \mathrm{~b}$. Consequently, in case (3), the contact line slips and the shape of the meniscus relaxes into a more stable shape, and the crystals stick again. Due to the well-known "slip-and-stick" movement of the contact line in faster growth conditions, poor ordering and discontinuous films are formed. Such details of the contact line movement and the nature of the solution deposition of nanoparticle assemblies have been reported.[15, 16]

To overcome such problems and eliminate the slip-and-stick movement of the contact line, we envisioned that adding a polymer would increase the viscosity of the semiconductor solution. In order not to impact the growth of the single-crystalline thin film or alter the charge-transport characteristics of organic semiconductors at the interface, we selected an amorphous, polar, and insulating polymer, whose physical properties differ from commonly designed organic semiconductors composed of a robust highly extended $\pi$-electron core and non-polar flexible long alkyl chains. As a result, the slip-and-stick movement of the contact 
line is prevented and the two-dimensional large single-crystalline organic semiconducting film is continuously formed as expected in case (2).

We hypothesized the effect and behavior of such a polymer in the continuous edge-casting method to be as follows (see Figure 1c). First, while evaporating the solvent, the polar polymer and the non-polar semiconductor begin to segregate, forming a bilayer thin film. Unlike crystal growth without a polymer, the viscous region is near the contact line. The solution sticks strongly to the substrate, preventing contact-line slippage, which is detrimental for continuous crystal growth. If such an insulating polymer is employed, the polymer layer should serve as a dielectric on the $\mathrm{SiO}_{2}$ and polymer substrate, allowing top-contact bottom-gate transistors to be fabricated by depositing gold on top of the thin film.

As the first trial of our solution process, we chose poly(methyl methacrylate) (PMMA) as a commercially available polymer. The difference in the physical properties between the alkyl-substituted highly extended organic semiconductor, $\mathbf{C}_{\mathbf{1 0}}-\mathbf{D N B D T}-\mathbf{N W}$, and PMMA should be immiscible in the film, leading to an effective phase separation, forming a clear interface between the semiconductor and the polymer.

Controlling the interface is the most important issue for the fast conduction of carriers in field-effect transistors. In this communication, we demonstrate the reliability and processability of the continuous edge-casting method using $\mathbf{C}_{\mathbf{1 0}}-\mathbf{D N B D T}-\mathbf{N W}$ and PMMA. Single-crystalline films of $\mathbf{C}_{\mathbf{1 0}}$-DNBDT-NW semiconductors are stably grown on the PMMA layer over a large area by effective phase separation at a crucially faster processing speed compared to the case without the addition of a polymer. Furthermore, we also show the limitations of two-dimensional continuous growth of a single-crystalline film in terms of the solution technique. However, it is noteworthy that the single-crystalline films of $\mathbf{C}_{\mathbf{1 0}}-$ DNBDT-NW / PMMA exhibit the highest and average mobilities of 17 and $10.6 \mathrm{~cm}^{2} / \mathrm{Vs}$, respectively. 


\section{Materials, substrates, device fabrications, and evaluations}

\subsection{Materials and substrates}

Organic semiconductor materials of $\mathbf{C}_{\mathbf{1 0}}$-DNBDT-NW were synthesized according to the previously reported method.[14] The insulator polymer, poly(methylmethacrylate) (PMMA) (average Mw 120,000 by GPC), was purchased from Sigma-Aldrich. As a substrate to fabricate the transistor, we used $n$-doped silicon wafers with a thermally oxidized layer as gate dielectrics.

\subsection{Device fabrication}

For the continuous edge-casting method, $\mathbf{C}_{\mathbf{1 0}}-\mathbf{D N B D T}-\mathbf{N W}$ and PMMA were dissolved in 3-chlorothiophene at a concentration of $0.025 \mathrm{wt} \%$ by heating to $100{ }^{\circ} \mathrm{C}$. The solution was supplied to the edge of the blade, which was located above the substrate on the hot plate (Figure 1a). To ensure continuous growth of the semiconductor thin films, the substrate was moved toward the direction depicted in Figure 1a. The solution supply rate was controlled to keep a certain amount of the solution at the edge of the blade. After crystallization, the thin films were heated in a vacuum at a temperature of $80{ }^{\circ} \mathrm{C}$ for 12 hours to remove the residual solvent incorporated in the films.

\subsection{Evaluations}

The Smart Lab of the Rigaku Cooperation was used for the out-of-plane X-ray diffraction measurements. A 191R diffractometer (Rigaku Cooperation) was used for the transmission X-ray diffraction measurements. For each XRD measurement, $\mathrm{Cu} K_{\alpha}$ radiation $(\lambda$ $=1.5418 \AA$ ) was used as the light source. A Keithley SCS4200 semiconductor parameter analyzer was used for the electrical measurements of the transistors. To analyze the mobility from the transfer curves, the actual capacitance of the binary dielectrics of the $\mathrm{SiO}_{2}$ and the PMMA layer was measured by $\mathrm{CV}$ measurements in accordance with the procedure described in the supporting information. 


\section{Results and discussion}

First, we prepared a thin film of $\mathbf{C}_{\mathbf{1 0}}-\mathbf{D N B D T}-\mathbf{N W}$ (Figure 1d) semiconductor solution with PMMA by the continuous edge-casting method with a substrate moving speed of 20,40 , or $80 \mu \mathrm{m} / \mathrm{s}$. Table 1 shows the cross-polarized optical microscope pictures of the films grown as well as the case without the addition of PMMA. In the case of a PMMA blend solution, the single-crystalline thin films of $\mathbf{C}_{\mathbf{1 0}}-\mathbf{D N B D T}-\mathbf{N W}$ are formed at all speeds from 20 to $80 \mu \mathrm{m} / \mathrm{s}$. On the other hand, in the case of only the $\mathbf{C}_{\mathbf{1 0}}-\mathbf{D N B D T}-\mathbf{N W}$ semiconductor solution, such thin films are only obtained at a speed of $20 \mu \mathrm{m} / \mathrm{s}$ in this solution processing condition (Table 1). The reflection light under the cross-polarized optical microscope is extinguished abruptly each time the sample is rotated, indicating that the size of the single-crystal domain exceeds a few hundred $\mu \mathrm{m}^{2}$ (Figure 1e). This result clearly demonstrates that the addition of a polymer to a semiconductor solution significantly affects the stable growth of single-crystalline films over a wide processing range in this polymer-blended solution process.

To analyze the thin films obtained from a $\mathbf{C}_{\mathbf{1 0}}-\mathbf{D N B D T}-\mathbf{N W}$ and PMMA blend, we evaluated its structural composition, surface, and thickness by the optical microscopy, cross-polarized optical microscopy, and atomic-force microscopy (AFM), respectively. Orthogonal solvents are used to selectively dissolve the component and reveal the film structure. Figures $2 \mathrm{a}$ and $2 \mathrm{~b}$ show the optical and cross-polarized optical microscope view of the thin film grown at $80 \mu \mathrm{m} / \mathrm{s}$ after the procedure to dissolve the component, respectively. The details of the procedure are as follows. First, the substrate is partially immersed into cyclohexane heated at elevated temperature $\left(80^{\circ} \mathrm{C}\right)$, which dissolves $\mathbf{C}_{\mathbf{1 0}}-\mathbf{D N B D T}-\mathbf{N W}$ moderately but not PMMA. This process corresponds to region II in Figures 2a and $2 \mathrm{~b}$. Second, the substrate is immersed into butyl acetate to dissolve PMMA; this step exposes the $\mathrm{SiO}_{2}$ surface (region I, Figures $2 \mathrm{a}$ and $2 \mathrm{~b}$ ). Under the cross-polarized optical microscope, the bright (region III, Figure 2b) and dark (region II, Figure 2b) areas correspond to the 
crystalline $\mathbf{C}_{\mathbf{1 0}}-\mathbf{D N B D T}-\mathbf{N W}$ film and the amorphous PMMA film, respectively. Figure 2c plots the height of the profile and clearly reveals two separated layers, each of which are about $10-20 \mathrm{~nm}$ thick.

Judging from the above procedure, the top and bottom layers are $\mathbf{C}_{\mathbf{1 0}}-\mathbf{D N B D T}-\mathbf{N W}$ and PMMA, respectively, which is why we propose a phase separation between them (Figure 1c). The phase separation behavior agrees well with previous reports by other groups using a combination of another semiconductor and PMMA to form binary films by spin-coating[17-19] and the blade-coating technique[20]. In the case of region II, Figure $2 d$ shows the morphology measured by AFM. The root-mean square (RMS) calculated from the variation of the height in the surface profile is $\sim 0.18 \mathrm{~nm}$, which is much smaller than the molecular length of $\mathbf{C}_{\mathbf{1 0}}-\mathbf{D N B D T}-\mathbf{N W}(4.1 \mathrm{~nm})$ (Figure 2e). This indicates that the surface is almost molecularly flat and differs from the recent reports with a one order of magnitude larger value.[20] Similar trends are obtained for processing speeds of 20 and $40 \mu \mathrm{m} / \mathrm{s}$, the result suggests that a well-defined interface between the semiconductor crystal and polymer amorphous layers is successfully formed during the continuous edge-casting process.

We also investigated the crystal structure, crystallinity, and molecular ordering of the $\mathbf{C}_{10}-\mathbf{D N B D T}-\mathbf{N W}$ crystalline layer by X-ray diffraction (XRD) measurements prepared at a processing speed of $80 \mu \mathrm{m} / \mathrm{s}$. In the out-of-plane scan, a series of peaks corresponding to $(h 0$ 0 ) in the bulk crystal structure (Figures $2 \mathrm{~h}-\mathrm{j}$ ) are observed (Figure $2 \mathrm{f}$ ), clearly indicating that the $a^{*}$ axis of the crystal is perpendicular to the substrate and the conduction $b-c$ plane is parallel to the substrate. In the transmission XRD, latticed Laue spots corresponding to a $b^{*}$ $c^{*}$ plane are seen (Figure $2 \mathrm{~g}$ ), indicating that the thin film is a single crystal irradiated within the area of the X-ray $\left(80 \mu \mathrm{m}^{2}\right)$. The aggregated structure in the layers is identical to the reported structure of $\mathbf{C}_{\mathbf{1 0}}-\mathbf{D N B D T}-\mathbf{N W}[14]$ and the latticed Laue spots demonstrate the high crystallinity of the $\mathbf{C}_{\mathbf{1 0}}-\mathbf{D N B D T}-\mathbf{N W}$ layers. 
Finally, we estimated the mobility of the obtained $\mathbf{C}_{\mathbf{1 0}}-\mathbf{D N B D T}-\mathbf{N W}$ single-crystalline PMMA binary thin film. The transistors were fabricated based on the bottom-gate-top-contact configuration (Figure 3a). The substrate was a $\mathrm{Si}$ wafer with thermally oxidized $\mathrm{SiO}_{2}(100 \mathrm{~nm})$, and the $\mathrm{SiO}_{2}$ layer played the role of gate dielectrics. After growing thin films on the substrate, a 50-nm thick gold electrode was evaporated in a vacuum through a shadow mask. Taking into account that the PMMA layer, which forms between the $\mathbf{C}_{10}-$ DNBDT-NW semiconductor crystal and the $\mathrm{SiO}_{2}$ substrate and partly serves as the gate dielectrics, the combined capacitance of the PMMA and $\mathrm{SiO}_{2}$ layers was estimated (see supporting information, Figure S1). Thus, the carriers accumulate at the interface between the $\mathbf{C}_{10}-\mathbf{D N B D T}-\mathbf{N W}$ crystal and the PMMA layer.

Figures $3 \mathrm{~b}-\mathrm{d}$ show the transistor characteristics for the thin film grown at $80 \mu \mathrm{m} / \mathrm{s}$. The present transistors have a mobility as high as $17 \mathrm{~cm}^{2} / \mathrm{Vs}$. It should be noted that this mobility is comparable to that reported for thin-film devices grown without PMMA.[14] This result suggests that the mobility itself is not impaired in the binary structure formed by the phase separation of $\mathbf{C}_{\mathbf{1 0}}-\mathbf{D N B D T}-\mathbf{N W}$ crystalline semiconductors and the PMMA amorphous polymer during semiconductor crystallization.

Figure 2e plots the average mobility of the thin film fabricated as a function of the substrate moving speed. The thin film crystallized from the $\mathbf{C}_{\mathbf{1 0}}-\mathbf{D N B D T}-\mathbf{N W}$ and PMMA blend solution does not show a mobility decrease, whereas the thin film crystallized from the $\mathbf{C}_{10}$-DNBDT-NW solution has a remarkable mobility drop. Using the case of the thin-film growth at a speed of $80 \mu \mathrm{m} / \mathrm{s}$ as an example, all eight devices exhibit a mobility exceeding 5 $\mathrm{cm}^{2} / \mathrm{Vs}$ in the thin films crystallized from the $\mathbf{C}_{\mathbf{1 0}}-$ DNBDT-NW and PMMA solution. In contrast none of the eight devices have a mobility over $10^{-2} \mathrm{~cm}^{2} / \mathrm{Vs}$ in the thin films crystallized from the $\mathbf{C}_{\mathbf{1 0}}$-DNBDT solution. These results clearly indicate that the addition of PMMA in the semiconductor solution helps widen the processing window to grow a single-crystalline thin film in our crystal growth process. 
Judging from the experiments above, the addition of a polymer with amorphous, polar, and insulating characteristics that differ from the properties of the semiconductor solution is a key strategy in stabilizing large-area single-crystal growth. Furthermore, using a combination of our solution method and blend solution, a large-area single-crystalline film measuring as large as $10 \times 10 \mathrm{~cm}^{2}$ can be grown at processing speed as high as $80 \mu \mathrm{m} / \mathrm{s}$. Amassian groups recently reported transistors fabricated by blade coating of a 2,8-difluoro-5,11-bis(triethylsilylethynyl)anthradithiophene (diF-TES-ADT): polystyrene (PS) blend solution with one or two orders of magnitude faster processing speeds. Although they demonstrated that the blend films exhibit a device performance on par with the case of diF-TES-ADT single crystal, the performance is below the minimum requirement for high-speed organic semiconductor TFTs $\left(10 \mathrm{~cm}^{2} / \mathrm{Vs}\right)$. Furthermore, the device yield is low due to the poorer quality of thin film in the fast process compared to the case of our single-crystalline film.

Considering the previous studies and our results, we argue that in addition to the development of a solution-processing technique, the development of organic semiconductors themselves together and organic solvents optimized for the process are both essential for very high performance OFET operations. Ideally two-dimensional continuously grown large-domain single-crystalline films at relatively low processing speeds will indeed exhibit the highest device performance with pronounced yields.

\section{Conclusion}

In summary, we developed an effective and steady solution-processing technique for a small molecule-type semiconductor, $\mathbf{C}_{\mathbf{1 0}}-\mathbf{D N B D T}-\mathbf{N W}$, by adding an amorphous PMMA polymer to produce stable growth of a two-dimensional large-area single-crystalline thin film. During the film formation, the mixture spontaneously segregates, forming a bilayer film composed of a single-crystalline semiconductor layer and an amorphous polymer layer. The 
continuous edge-casting method can fabricate $\mathbf{C}_{\mathbf{1 0}}-\mathbf{D N B D T}-\mathbf{N W}$ single-crystalline thin films without impairing their mobility around $17 \mathrm{~cm}^{2} / \mathrm{Vs}$, even when the processing speed is 80 $\mu \mathrm{m} / \mathrm{s}$, which is four times faster than the optimum crystal growth speed without a polymer.

\section{Acknowledgements}

This work was supported by the JST PRESTO program "Molecular Technology and Creation of New Functions". J. S. acknowledges the JSPS research fellowship for young scientists. T. O. thanks a JSPS Grant-in-Aid for Scientific Research (B) (No.25288091). C. M. thanks a JSPS Grant-in-Aid for Young Scientists (B) (No.25810118). 


\section{References}

[1] S.R. Forrest, Nature 428 (2004) 911-918.

[2] G.H. Gelinck, H.E.A. Huitema, E. van Veenendaal, E. Cantatore, L. Schrijnemakers, J.B.P.H. van der Putten, T.C.T. Geuns, M. Beenhakkers, J.B. Giesbers, B.-H. Huisman, E.J. Meijer, E.M. Benito, F.J. Touwslager, A.W. Marsman, B.J.E. van Rens, D.M. de Leeuw, Nat. Mater. 3 (2004) 106-110.

[3] B. Crone, A. Dodabalapur, Y.Y. Lin, R.W. Filas, Z. Bao, A. LaDuca, R. Sarpeshkar, H.E. Katz, W. Li, Nature 403 (2000) 521-523.

[4] E. Cantatore, T.C.T. Geuns, G.H. Gelinck, E. van Veenendaal, A.F.A. Gruijthuijsen, L. Schrijnemakers, S. Drews, D.M. de Leeuw, IEEE Journal of Solid-State Circuits 42 (2007) 84-92.

[5] H.E. Katz, Chem. Mater. 16 (2004) 4748-4756.

[6] A.C. Arias, J.D. MacKenzie, I. McCulloch, J. Rivnay, A. Salleo, Chem. Rev. 110 (2010) $3-24$.

[7] M. Mas-Torrent, C. Rovira, Chem. Soc. Rev. 37 (2008) 827-838.

[8] S. Allard, M. Forster, B. Souharce, H. Thiem, U. Scherf, Angew. Chem. Inter. Ed. 47 (2008) 4070-4098.

[9] H. Minemawari, T. Yamada, H. Matsui, J.y. Tsutsumi, S. Haas, R. Chiba, R. Kumai, T. Hasegawa, Nature 475 (2011) 364-367.

[10] G. Giri, E. Verploegen, S.C.B. Mannsfeld, S. Atahan-Evrenk, D.H. Kim, S.Y. Lee, H.A. Becerril, A. Aspuru-Guzik, M.F. Toney, Z. Bao, Nature 480 (2011) 504-508.

[11] J. Takeya, M. Yamagishi, Y. Tominari, R. Hirahara, Y. Nakazawa, T. Nishikawa, T. Kawase, T. Shimoda, S. Ogawa, Appl. Phys. Lett. 90 (2007) 102120-102123.

[12] T. Uemura, Y. Hirose, M. Uno, K. Takimiya, J. Takeya, Appl. Phys. Express 2 (2009) 111501. 
[13] J. Soeda, T. Uemura, T. Okamoto, C. Mitsui, M. Yamagishi, J. Takeya, Appl. Phys. Express 6 (2013) 076503.

[14] C. Mitsui, T. Okamoto, M. Yamagishi, J. Tsurumi, K. Yoshimoto, K. Nakahara, J. Soeda, Y. Hirose, H. Sato, A. Yamano, T. Uemura, J. Takeya, Adv. Mater. 26 (2014) 4546-4551.

[15] E. Adachi, A.S. Dimitrov, K. Nagayama, Langmuir 11 (1995) 1057-1060.

[16] R.D. Deegan, O. Bakajin, T.F. Dupont, G. Huber, S.R. Nagel, T.A. Witten, Nature 389 (1997) 827-829.

[17] A. Kumatani, C. Liu, Y. Li, P. Darmawan, K. Takimiya, T. Minari, K. Tsukagoshi, Sci. Rep. 2 (2012) 393.

[18] C. Liu, Y. Li, T. Minari, K. Takimiya, K. Tsukagoshi, Org. Electron. 13 (2012) 1146-1151.

[19] X. Wang, W.H. Lee, G. Zhang, X. Wang, B. Kang, H. Lu, L. Qiu, K. Cho, J. Mater. Chem. C 1 (2013) 3989-3998.

[20] M.R. Niazi, R. Li, E. Qiang Li, A.R. Kirmani, M. Abdelsamie, Q. Wang, W. Pan, M.M. Payne, J.E. Anthony, D.-M. Smilgies, S.T. Thoroddsen, E.P. Giannelis, A. Amassian, Nat. Commun. 6 (2015) 8598. 
Table 1. Cross-polarized optical microscope pictures of crystalline thin films of $\mathbf{C}_{\mathbf{1 0}}$ DNBDT-NW crystallized in different conditions.

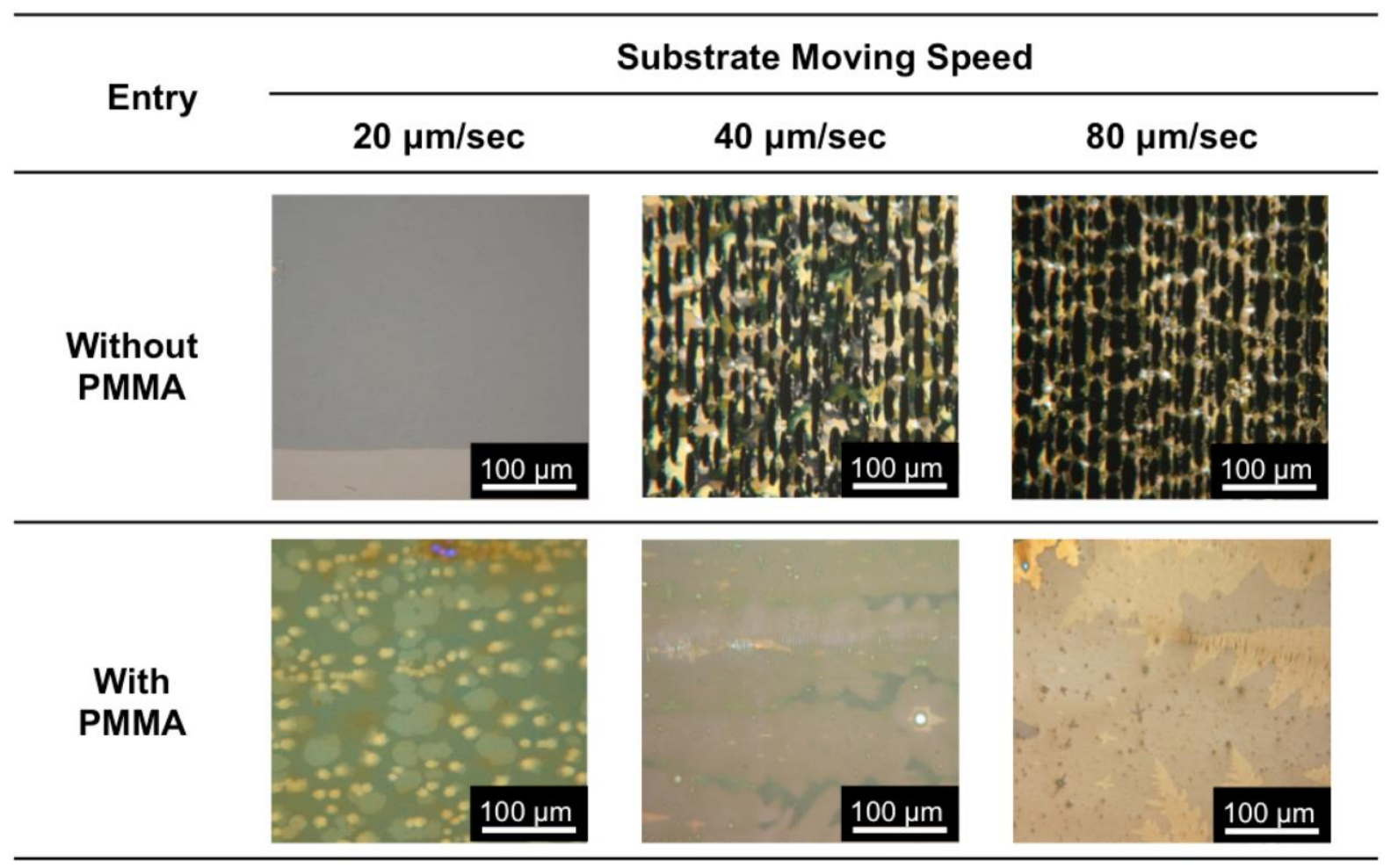




\section{(a)}
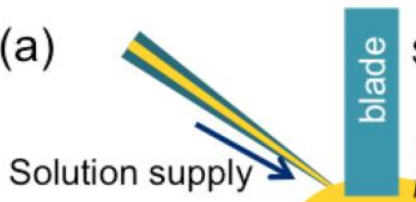

Solution

Substrate

(c)

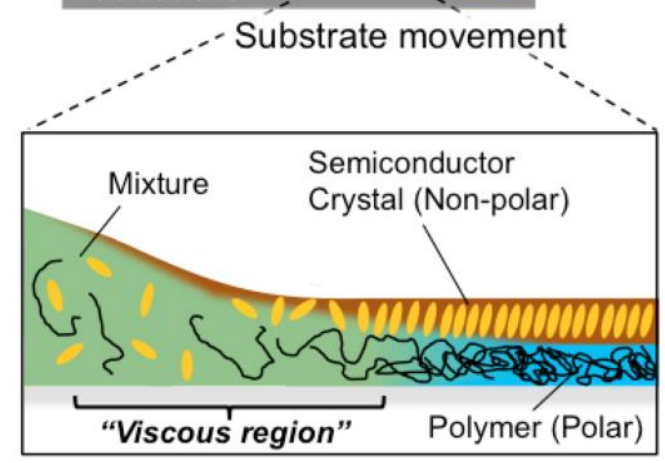

(b)

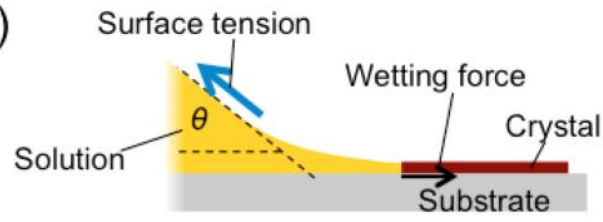

case 2

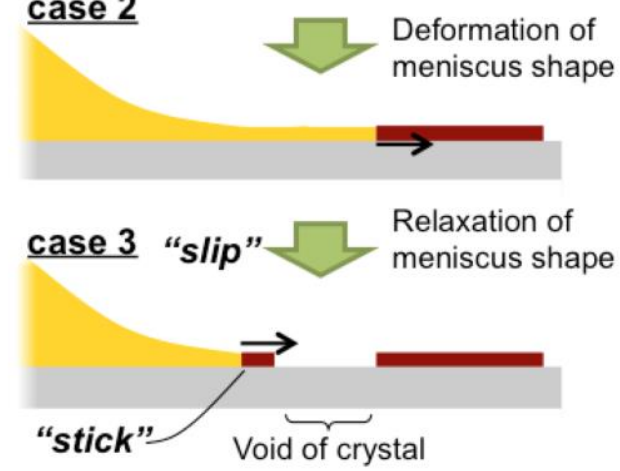

(d)

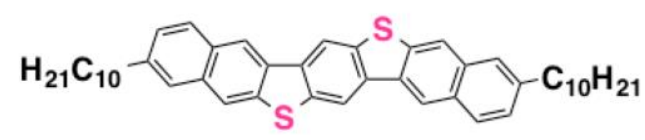

3,11-didecyldinaphtho[2,3-d:2',3'-d'] benzo[1,2- $\left.b: 4,5-b^{\prime}\right]$ dithiophene $\left(\mathrm{C}_{10}\right.$-DNBDT-NW) (e)

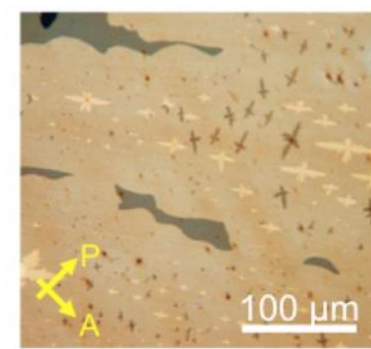

Figure 1. (a) Schematics of the continuous edge-casting method. (b) Schematics of the growth instability. (c) Magnified figure of the contact region describing the mechanism of crystal-growth stabilization with a polymer additive. (d) Molecular structure of $\mathbf{C}_{\mathbf{1 0}}-\mathbf{D N B D T}$ NW. (e) Cross-polarized optical microscopic picture of the thin film grown from a polymer/semiconductor blend solution. Crystalline thin film is grown at a speed of $80 \mu \mathrm{m} / \mathrm{s}$. 

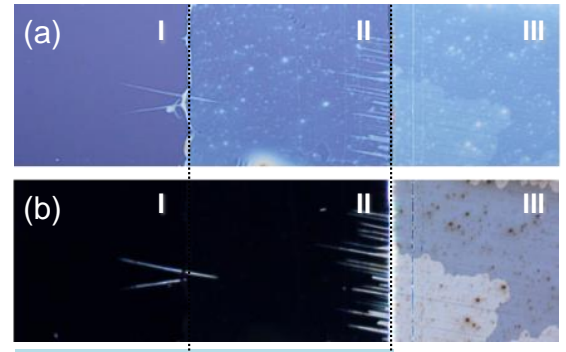

Cyclohexane
Butyl acetate
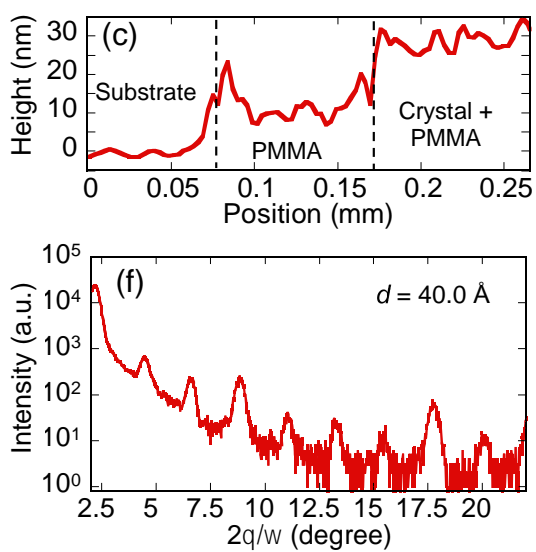
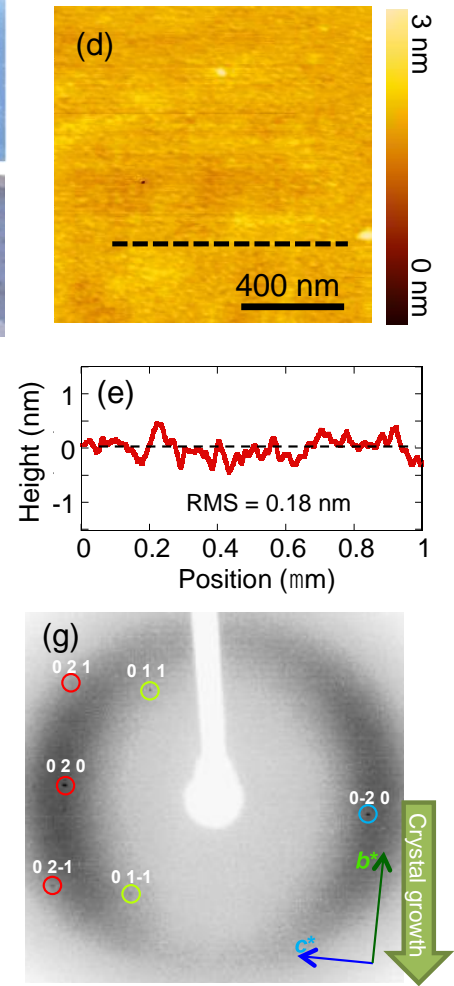

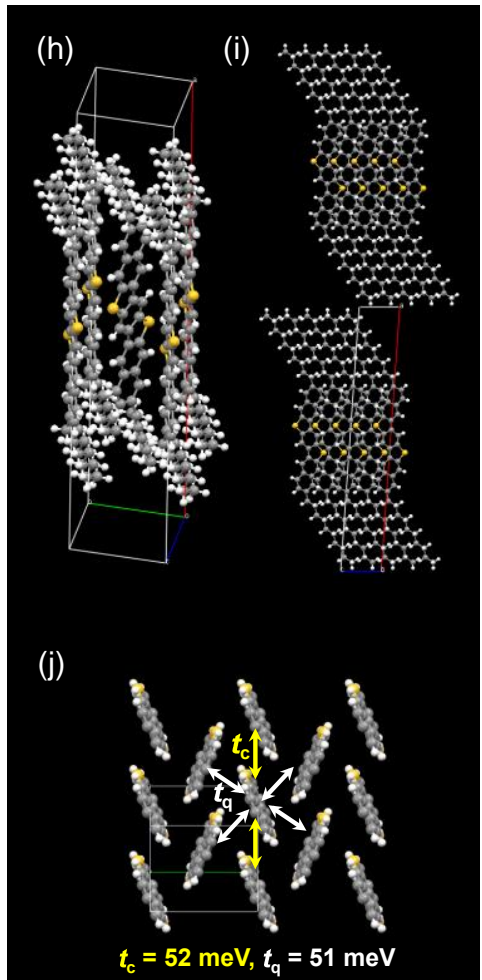

Figure 2. (a) Optical microscope and (b) cross-polarized optical microscope view of a thin film grown at a speed of $80 \mu \mathrm{m} / \mathrm{s}$ from a $\mathbf{C}_{\mathbf{1 0}}$-DNBDT-NW /PMMA blend solution (region III). $\mathbf{C}_{\mathbf{1 0}}-\mathbf{D N B D T}-\mathbf{N W}$ crystalline film is partially removed by cyclohexane, exposing the PMMA layer (region II). Exposed PMMA layer is also partially removed by the butyl acetate (region I). Bars displayed below the pictures indicate the area where the film is dipped in the respective solvent. (c) Typical surface profile of the thin film. (d) Atomic force microscopy image of the surface of PMMA after removal of the $\mathbf{C}_{\mathbf{1 0}}-\mathbf{D N B D T}-\mathbf{N W}$ crystalline film by cyclohexane and (e) the surface profile (dashed line). (f) Diffraction profile of the out-of-plane XRD measurement. (g) Diffraction pattern of the transmission XRD measurement. ( $\mathrm{h}-\mathrm{j}$ ) Crystal structure of $\mathbf{C}_{\mathbf{1 0}}-\mathbf{D N B D T}-\mathbf{N W}$ viewed from $(\mathrm{h})$ a bird-eye's of a unit cell, (i) along the $b$-axis, and (j) along the $a^{*}$-axis with transfer integral values calculated by the PW-91/TZP level in the ADF program. Alkyl chains are omitted for clarify. 
(a)
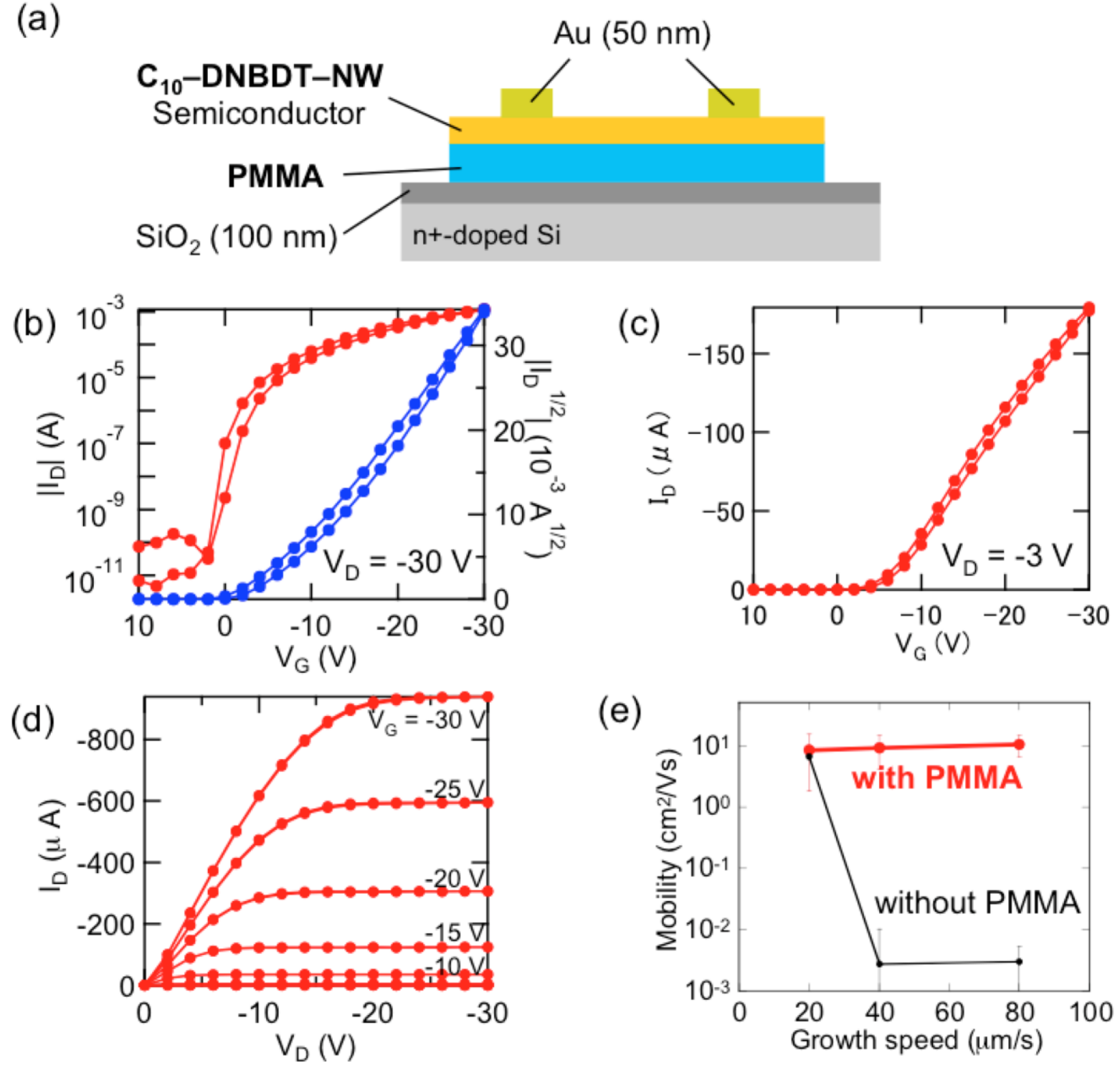

Figure 3. (a) Device structures in the bottom-gate-top-contact configuration. (b-d) Transistor characteristics of a TFT fabricated with the present thin films grown from a polymer/semiconductor blend solution. Crystalline thin film grown at a speed of $80 \mu \mathrm{m} / \mathrm{s}$. (b, c) Transfer characteristics at (b) saturation and (c) the linear regime. (d) Output characteristics of the present transistor. (e) Crystal growth speed dependence of the average mobility of the TFTs. Each point and error bar represent the average mobility and standard deviation among the ten devices fabricated under the same crystal growth conditions, respectively. Thin films are grown from a PMMA/C $\mathbf{C}_{\mathbf{1 0}}-\mathbf{D N B D T}-\mathbf{N W}$ blend solution (red line) and a $\mathbf{C}_{\mathbf{1 0}}-\mathbf{D N B D T}-$ NW solution without PMMA (black line). 

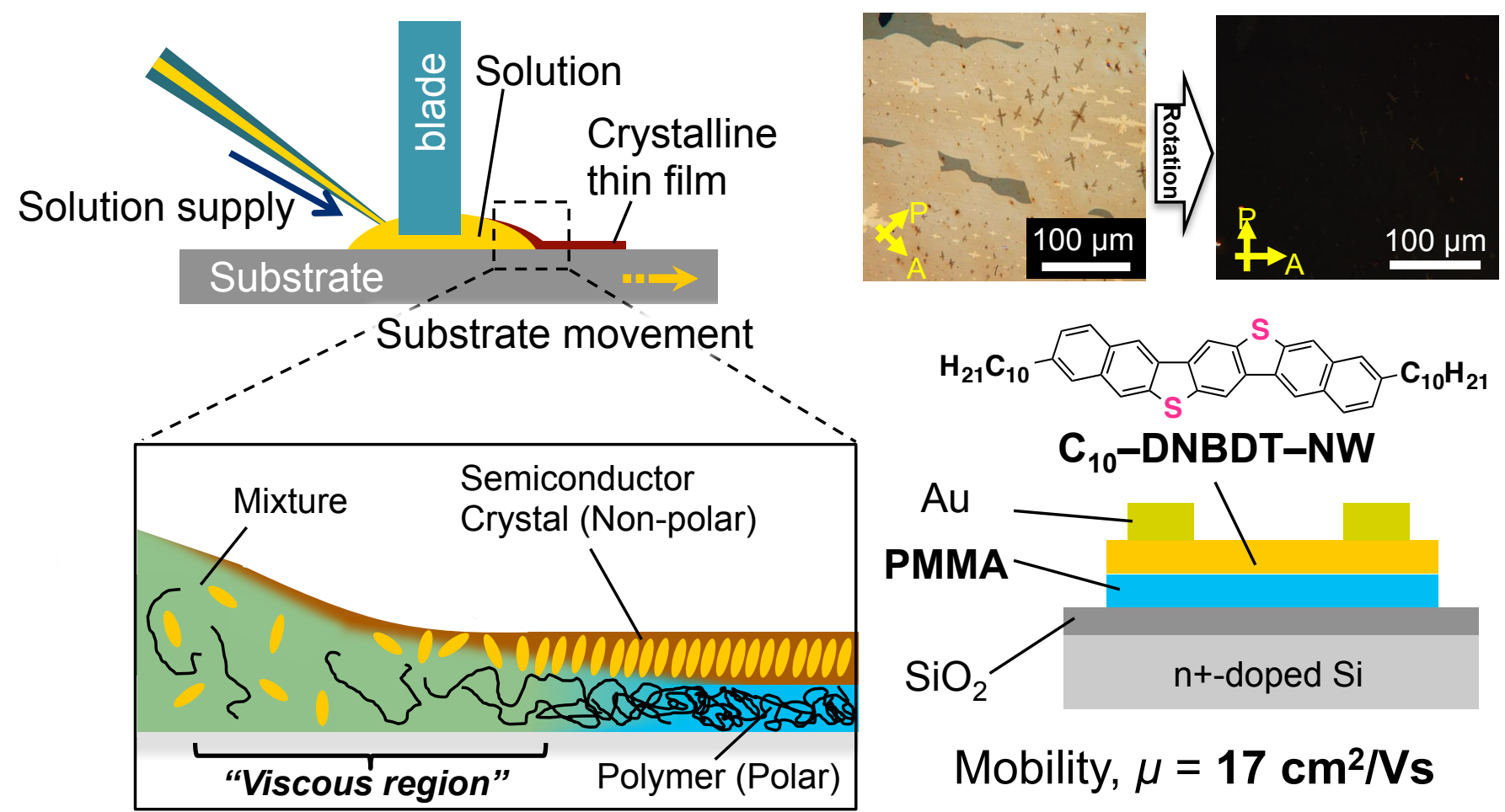

Mobility, $\mu=17 \mathrm{~cm}^{2} / \mathrm{Vs}$ 